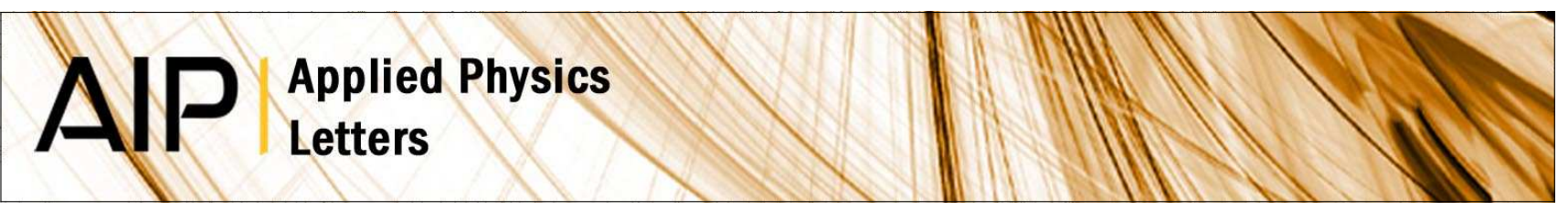

\title{
Influence of a depletion layer on localized surface waves in doped semiconductor nanostructures
}

S. Vassant, F. Pardo, P. Bouchon, R. Haïdar, F. Marquier et al.

Citation: Appl. Phys. Lett. 100, 091103 (2012); doi: 10.1063/1.3689747

View online: http://dx.doi.org/10.1063/1.3689747

View Table of Contents: http://apl.aip.org/resource/1/APPLAB/v100/i9

Published by the American Institute of Physics.

\section{Related Articles}

A pancake-shaped nano-aggregate for focusing surface plasmons

J. Appl. Phys. 111, 034308 (2012)

Plasmon assisted light propagation and Raman scattering hot-spot in end-to-end coupled silver nanowire pairs Appl. Phys. Lett. 100, 043108 (2012)

Energy-filtered phase retrieval using the transport of intensity equation

Appl. Phys. Lett. 99, 221905 (2011)

Scaling of losses with size and wavelength in nanoplasmonics and metamaterials

Appl. Phys. Lett. 99, 211106 (2011)

Collective excitations of electron gas on the nanotube surface in a magnetic field

Low Temp. Phys. 37, 919 (2011)

\section{Additional information on Appl. Phys. Lett.}

Journal Homepage: http://apl.aip.org/

Journal Information: http://apl.aip.org/about/about_the_journal

Top downloads: http://apl.aip.org/features/most_downloaded

Information for Authors: http://apl.aip.org/authors

\section{ADVERTISEMENT}

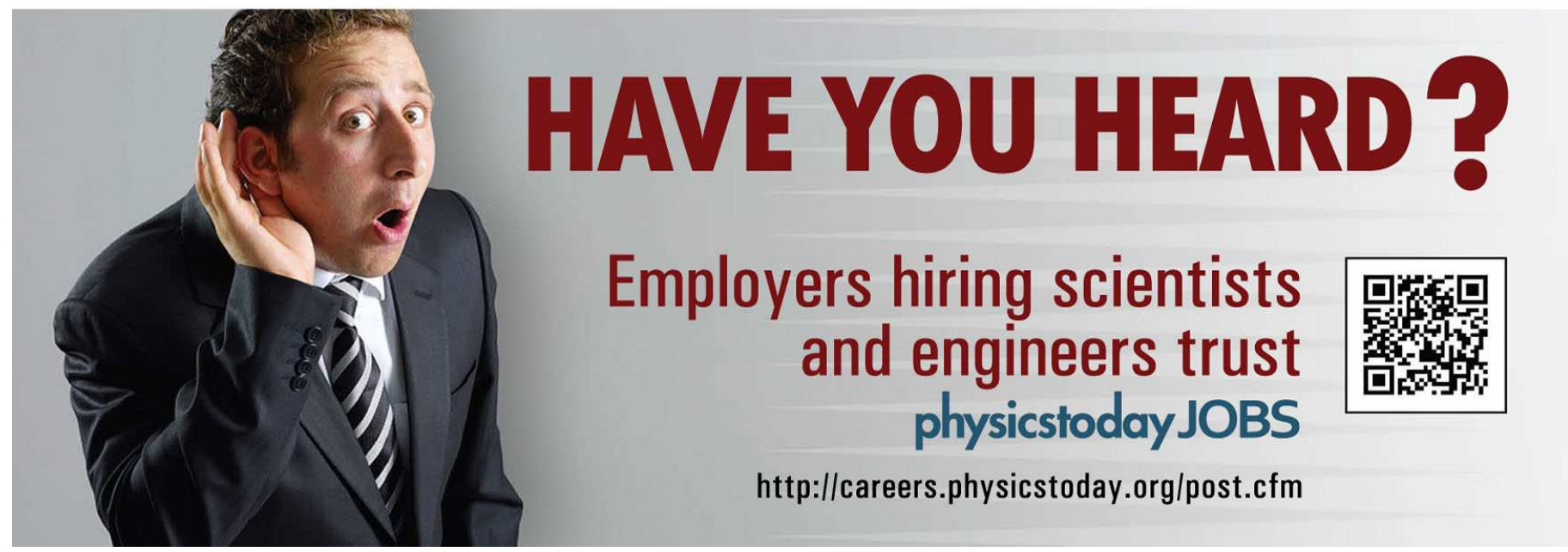




\title{
Influence of a depletion layer on localized surface waves in doped semiconductor nanostructures
}

\author{
S. Vassant, ${ }^{1,2, a)}$ F. Pardo, ${ }^{1}$ P. Bouchon, ${ }^{3}$ R. Haïdar, ${ }^{3}$ F. Marquier, ${ }^{2}$ J. J. Greffet, ${ }^{2}$ \\ and J. L. Pelouard ${ }^{1}$ \\ ${ }^{1}$ Laboratoire de Photonique et Nanostructures (LPN-CNRS), Route de Nozay, F-91460 Marcoussis, France \\ ${ }^{2}$ Laboratoire Charles Fabry, Institut d'Optique, CNRS, Univ. Paris-Sud, 2 avenue Augustin Fresnel, 91127 \\ Palaiseau cedex, France \\ ${ }^{3}$ ONERA - The French Aerospace Lab, F-91761 Palaiseau, France
}

(Received 18 October 2011; accepted 7 February 2012; published online 27 February 2012)

\begin{abstract}
We report $\mathrm{THz}$ polarized reflectivity measurements on a doped GaAs grating made of thin walls with subwavelength dimensions and periodicity. A clear dip appears in the TM-polarized reflectivity spectrum, due to the excitation of a localized surface plasmon-phonon mode along the grating walls. The theoretical model used to describe the electromagnetic response of the grating shows the importance of the depletion layer at the GaAs surface and indicates a high sensitivity of the reflectivity dip frequency to the surface potential. (C) 2012 American Institute of Physics. [http://dx.doi.org/10.1063/1.3689747]
\end{abstract}

With the increasing research activity in the $\mathrm{THz}$ field, there is a renewed interest in surface waves supported by semiconductors (SCs). Two types of surface waves can propagate on a SC: surface phonons polaritons $(\mathrm{SPhP})$ in the reststrahlen band for polar SC and surface plasmons polaritons (SPP) if the SC (polar and not polar) is doped. When the electron plasma frequency in the $\mathrm{SC}$ is close to the optical phonon frequencies, both surface waves couple leading to surface plasmon-phonon polaritons $(\mathrm{SPPhP}){ }^{1}$ Since the seventies, theoretical studies ${ }^{2}$ have predicted that the propagation of surface waves could be modified by the presence of a depletion or accumulation layer at the SC/air interface. For example, in the case of GaAs, the crystal symmetry breaking at the surface creates a distribution of levels in the gap of the SC that lead to the pinning of the Fermi level. ${ }^{3}$ This entails the formation of a carrier-depleted layer at the GaAs surface. From Ref. 2, the influence of this layer on surface waves is expected only at large wavevectors. Thus, most of the experimental efforts have been focused on electron energy loss spectroscopy to observe the effect of depletion (and accumulation) layers in SCs on surface waves (see Ref. 4 for example).

The influence of the depletion layer on optical measurements has been explored by infrared ellipsometry on flat surfaces (see Ref. 5 and reference therein), but there are only a few studies concerning structurated SC supporting surface waves. The reflectivity of doped SC structurated surfaces was measured in the seventies ${ }^{6,7}$ without reliable results because fabrication techniques were not as developed as nowadays. Both in Refs. 6 and 7, the fabrication process damaged the sample surface and the geometries of the samples were not well known. A depletion layer was invoked to explain discrepancies between experimental and theoretical results. Other studies can be found, where the transmission of small crystals embedded in a matrix is measured. Rieder et al. studied SPPhP modes of $\mathrm{CdO}$ microcystals ${ }^{8}$ and noted that an hypothetical non-uniform electron distribution in the

\footnotetext{
a) Author to whom correspondence should be addressed. Electronic mail: simon.vassant@institutoptique.fr.
}

crystals could have an effect on the spectra. Later, a coreshell model was proposed by Yamamoto et al., ${ }^{9}$ giving a qualitative agreement with the measurements of doped GaAs micro-crystals. However their size was not uniform and the shell thickness was only a phenomenological parameter, unrelated to the surface potential.

In this paper, we report experimental results on a lamellar doped SC grating made of parallel thin walls. Each wall has a width of the order of a few depletion layer thicknesses. TM-polarized reflectivity measurements show a dip that is due to the excitation of localized SPPhP modes. Comparison with theory indicates that the depletion layer has to be taken into account to fit the data. This dependance can be used to measure optically surface potential changes.

The geometry of the fabricated grating is schematically shown in Fig. 1(a). The fabrication process is the same as the first steps of Ref. 10. The grating lines are defined by e-beam lithography, followed by a Ti/Ni metallization and a lift-off. The metallic lines then serve as a mask for an inductively coupled plasma reactive ion etching with $\mathrm{BCl}_{3}, \mathrm{Cl}_{2}$, and Ar gases. The metallic mask is then removed in a diluted HF solution.

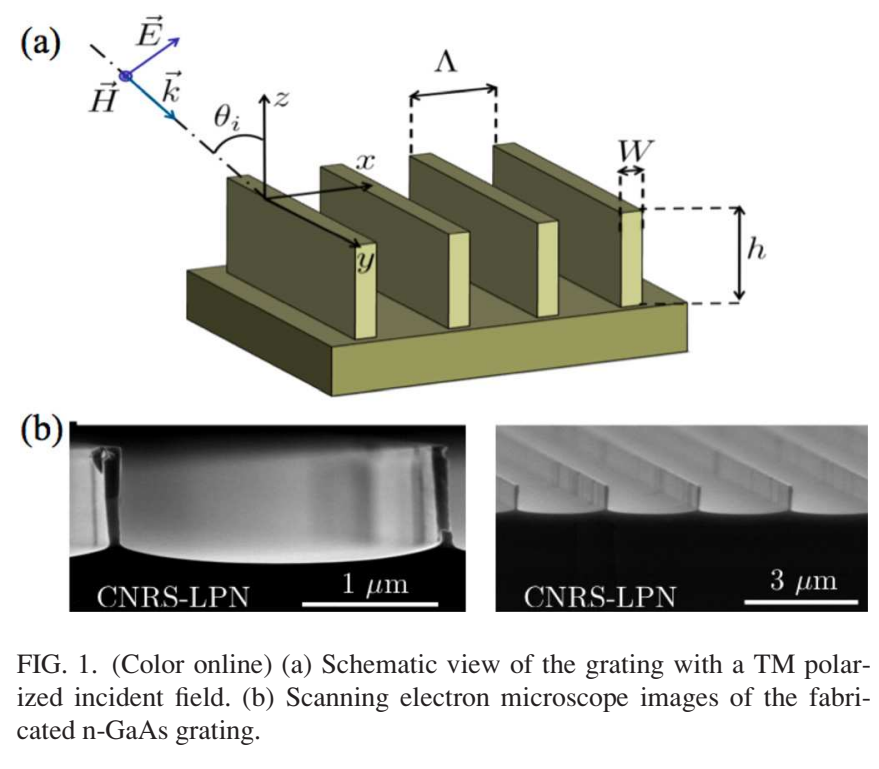


The etching process is strongly anisotropic as shown in the scanning electron microscope (SEM) cross-section images in Fig. 1(b). The grating period $\Lambda$ is $2.5 \mu \mathrm{m}$. From SEM pictures, we measure the wall width $(W=85 \mathrm{~nm})$ and height $(h=860 \mathrm{~nm})$.

The sample was placed in a Brucker Vertex 70 Fourier transform infrared spectrometer (FTIR), purged with dry air, equiped with an A-513Q reflectometry instrument, and a $\mathrm{THz}$ polarizer (Tydex). Reflectivity references were taken on a gold mirror (Ti/Au, 20/200 nm) deposited on the sample next to the grating. We used a HeNe laser beam impinging on the grating ensures the setup alignment: the observation of $-1,0$, and +1 diffraction orders allowed to define the plane of incidence with a precision of $3 \mathrm{mrad}$.

Reflectivity spectra of the sample (flat surface and grating) are measured in TM and TE polarization for different incident angles $\theta_{i}$ (Fig. 2).

All curves in Fig. 2 show a dip at $39.1 \mu \mathrm{m}$. This corresponds to an effect of the transverse optical phonon of GaAs on the dielectric function. A dip is observed in the reflectivity at $35.3 \mu \mathrm{m}$, only if the incident light is TM polarized. Its frequency does not change with incident angle, whereas its amplitude increases from 0.85 at $\theta_{i}=15^{\circ}$ (limit of the setup) to 0.41 at $\theta_{i}=50^{\circ}$. Note that at the same wavelength the reflectivity of the flat surface is 0.9 at $\theta_{i}=45^{\circ}$. The angular independence of the dip position indicates that we have observed a localized resonance. As detailed in the next section, the real part of the dielectric function of $\mathrm{n}$-doped GaAs is negative at $35.3 \mu \mathrm{m}$ due to optical phonon resonances of the polar lattice and free electron contribution. Hence, we attribute the $35.3 \mu \mathrm{m}$ absorption dip to the excitation of a localized SPPhP.

To confirm this hypothesis, we performed numerical simulations of the reflectivity spectrum using a rigorous maxwell calculation with constitutive approximation code (RMCA). ${ }^{11}$ This method allows using an adaptative mesh to describe the structure which is very appropriate to precisely mesh the thin grating walls. To describe the doped GaAs optical behavior, we use the following dielectric function: ${ }^{12}$

$$
\epsilon(\omega)=\epsilon_{\infty}\left(1-\eta_{\mathrm{ph}}-\eta_{e}\right),
$$

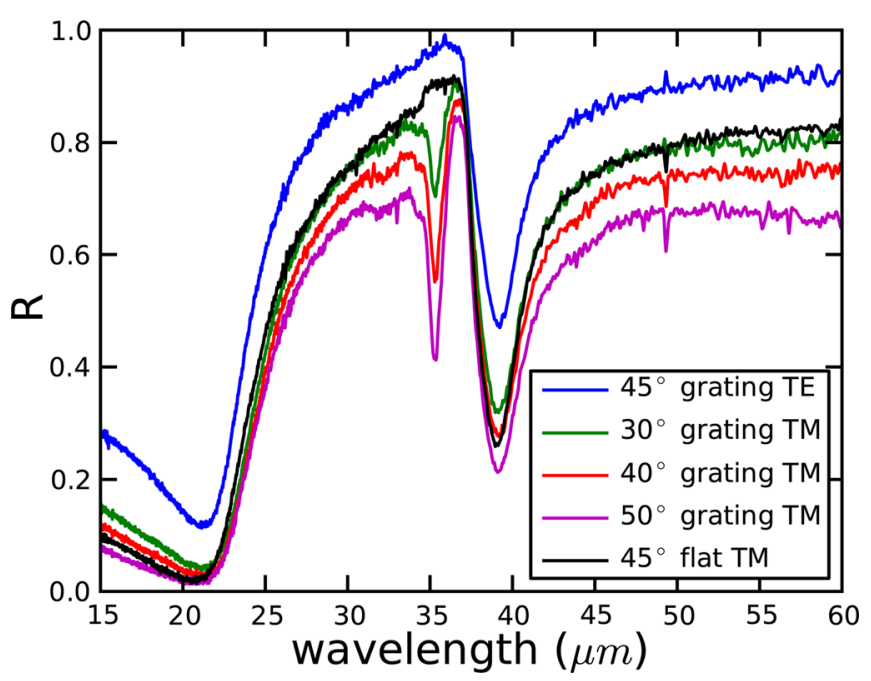

FIG. 2. (Color online) Experimental reflectivity spectra of the sample (flat surface and grating) measured at various angles of incidence in TM and TE polarization.

$$
\begin{gathered}
\eta_{\mathrm{ph}}=\frac{\omega_{L}^{2}-\omega_{T}^{2}}{\omega^{2}-\omega_{T}^{2}+i \Gamma \omega}, \\
\eta_{e}=\frac{\omega_{p}^{2}}{\omega^{2}+i \Gamma_{e} \omega},
\end{gathered}
$$

where $\eta_{\mathrm{ph}}$ corresponds to the polar lattice contribution and $\eta_{e}$ to the free electrons contribution. The frequencies $\omega_{T}$ and $\omega_{L}$ correspond, respectively, to the transverse and longitudinal optical phonons of the SC, $\Gamma$, and $\Gamma_{e}$ are the damping factors, respectively for phonons and electrons. The plasma frequency $\omega_{p}$ is given by $\omega_{p}^{2}=N_{D} e^{2} / \epsilon_{0} \epsilon_{\infty} m^{*}, N_{D}$ is the electron density, $e$ the electron charge, $\epsilon_{\infty}$ the high frequency dielectric constant of the SC, $\epsilon_{0}$ the vacuum permittivity, and $m^{*}$ the effective electronic mass. The phonon parameters $\left(\omega_{L}, \omega_{T}, \Gamma\right)$ were taken from the litterature. ${ }^{13}$ The carrier density $N_{D}$ and $\Gamma_{e}$ are determined by fitting a spectrum measured on the flat GaAs surface. We obtain $N_{D}=(1.47 \pm 0.01) \times 10^{18} \mathrm{~cm}^{-3}$ and $\Gamma_{e}=(69.5 \pm 0.1) \mathrm{cm}^{-1}$. As shown in a previous work, ${ }^{14}$ we have to take into account the curvature at the bottom of the structure due to the etching process (see Fig. 1(b)).

To compare the measurements with simulations, we start by considering two limiting cases: (1) the walls are uniformly occupied by electrons, i.e., without a depletion layer (Fig. 3(a)) and (2) the walls are fully depleted (Fig. 3(b)).

In the first case (Fig. 3(a)), the dip at $35.3 \mu \mathrm{m}$ is reproduced in the simulation, but with a large shift towards lower wavelengths and a much broader shape. In the second case (Fig. 3(b)), the dip at $35.3 \mu \mathrm{m}$ is again observed, but with a shift towards higher wavelength and a decrease in width. In

(a)

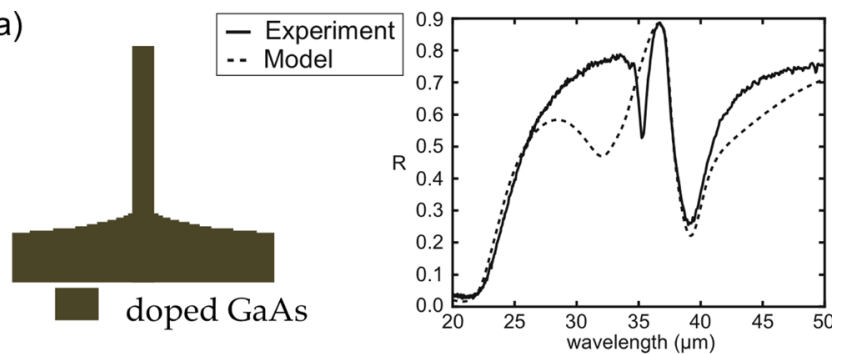

(b)
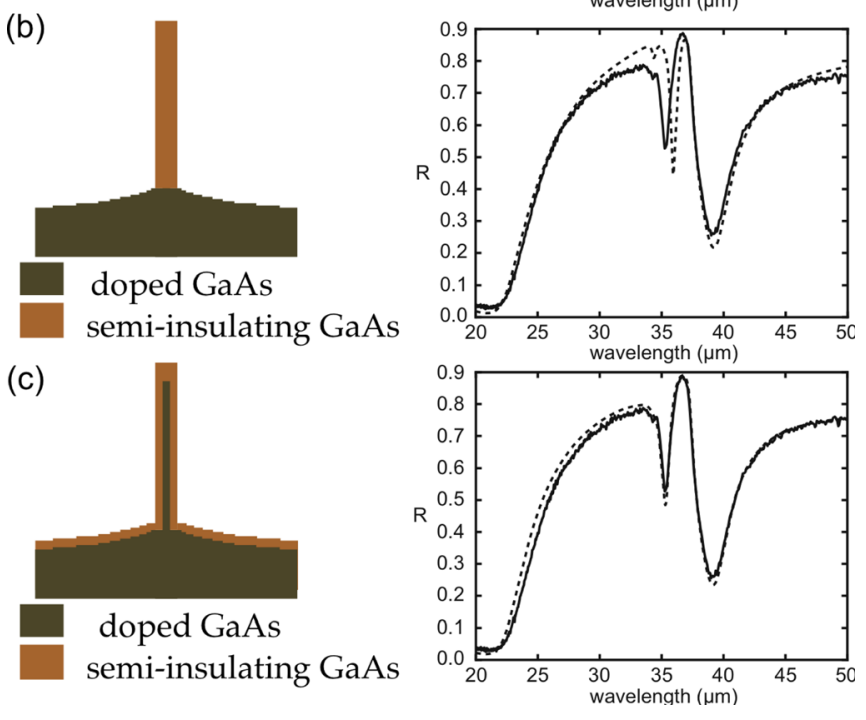

FIG. 3. (Color online) Comparison of the measured reflectivity of the GaAs grating $\left(\theta_{i}=45^{\circ}\right.$, TM polarized) with the theoretical reflectivity obtained with three models: (a) walls uniformly filled with electrons, (b) fully depeleted walls, and (c) "core-shell" model. 


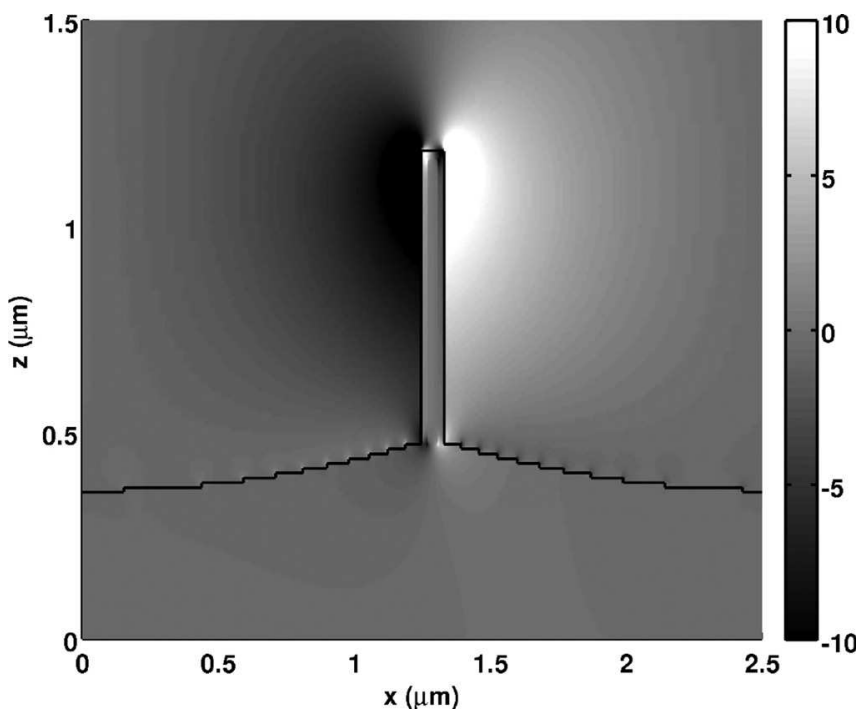

FIG. 4. Real part of the electric field along the $x$ direction at $35.3 \mu \mathrm{m}$ for $\theta_{i}=45^{\circ}$ and TM-polarization. One period is shown. The black line indicates the surface of the grating.

both cases, no satisfying agreement can be found by changing the geometric parameters in the limit of the SEM measurement precision $( \pm 10 \%)$.

We now consider a "core-shell" model as illustrated in Fig. 3(c), to take into account the depletion layer originating from the Fermi level pinning at the GaAs surface. We use the approximation of complete depletion to calculate the thickness $d$ of the shell: $d=\sqrt{2 V_{s} \epsilon_{0} \epsilon_{\text {stat }} / e N_{D}}$, where $\epsilon_{\text {stat }}$ is the static dielectric constant of the $\mathrm{SC}$ and $V_{s}$ the surface potential. For GaAs, $V_{s}$ is known to be relatively constant at $0.7 \mathrm{eV}^{3}$ giving $d \approx 26 \mathrm{~nm}$ for an electron density of $1.47 \times 10^{18} \mathrm{~cm}^{-3}$. The inner part of the wall has a dielectric constant given by Eq. (1) with $N_{D}$ and $\Gamma_{e}$ parameters identical to those of the substrate, while the outer shell is composed of fully depleted GaAs. With this model, we get an excellent agreement between theoretical and experimental reflectivities at $\theta_{i}=45^{\circ}$ (Fig. 3(c)) and for all the angles between $15^{\circ}$ and $50^{\circ}$ (not shown).

Using these extracted parameters at $35.3 \mu \mathrm{m}$, we have calculated the near field distribution in the structure to understand the nature of the mode excited in the grating. As seen on Fig. 4, the electric field is strongly concentrated along the vertical walls. Thus, the coupling between the walls is negligible. We can consider each wall of the grating as a nanoantenna. This agrees with the experimentally observed angular independence of the reflectivity dip frequency. The electric field component $E_{x}$ is replace antisymetric by antisymmetric and is similar to the short-range SPP mode supported by a thin metallic slab. ${ }^{15}$ This mode cannot be excited at normal incidence, and the coupling is an increasing function of the angle $\theta_{i}$. This explains our experimental observation of the dip amplitude variation with this angle. The short range SPP is known to have a noticeably larger effective index than those of the surrounding dielectric due to the coupling of two SPP through the metallic thin film. Here, the coupling of two SPPhP through a $85 \mathrm{~nm}$ thick wall leads to an increase of the effective index $n_{\text {eff }}$ by a factor close to seven. From our calculations we obtain $n_{\text {eff }}=7.54+2.17 i$ whereas the effective index for a flat interface separating air from undoped GaAs is
$1.092+0.026 \mathrm{i}$ and $1.016+0.004 \mathrm{i}$ for doped GaAs. Since each wall of the grating acts as a resonant nanoantenna, the resonant wavelength $\lambda_{r}$ is determined by the antenna length (here, the grating height $h$ ). As the geometry is similar to a ground-plane antenna, we expect a $\lambda / 4$ resonant behaviour. In fact, we obtain $\lambda_{r}=5.44 h n_{\mathrm{eff}}$ which can be explained by dephasing upon reflection at the bottom and top of the wall.

Finally, we evaluate the sensitivity of the SPPhP frequency to the model parameters. Changing the surface potential by $\pm 25 \mathrm{meV}$ produces a frequency shift of the reflectivity dip of $\pm 25 \mathrm{~nm}$, while its amplitude remains unchanged. A variation of only $1 \mathrm{~nm}$ in $W$ induces a shift of the resonance wavelength of about $34 \mathrm{~nm}$, while the amplitude is kept at the same value. The height of the grating has a lower influence compared to surface potential and wall thickness. A $1 \mathrm{~nm}$ variation in height $h$ induces both wavelength and amplitude changes of, respectively, $1.4 \mathrm{~nm}$ and $0.5 \%$. All these modifications only affect the reflectivity dip due to the SPPhP and do not alter the rest of the spectrum. The shape of the resonance itself is also preserved. These shifts can be measured by common FTIR techniques which mean that $\mathrm{THz}$ reflectivity on structurated polar materials can be used as a non-destructive method to probe surface potential variations.

To summarize, we studied by FTIR polarized reflectivity measurements localized SPPhPs on a one dimensional grating with very small wall widths. We have shown that the surface depletion layer plays an important role in the electromagnetic response of the grating. The introduction of a "core-shell" model allows a good description of the electromagnetic response of the grating. Using this model, it is possible to extract surface potential variations from the spectral reflectivity shifts with an accuracy of tens of $\mathrm{meV}$, which can be used for biosensing or surface chemistry studies.

This work has been supported by ANR contract LAPSUS and by the region Ile de France through a C' Nano Idf project. We thank N. Bardou, C. Dupuis, L. Ferlazzo, and E. Cambril for their help in the clean room.

${ }^{1}$ V. V. Bryksin, D. N. Mirlin, and I. I. Reshina, Solid State Commun. 11, 695 (1972).

${ }^{2}$ S. L. Cunningham, A. A. Maradudin, and R. F. Wallis, Phys. Rev. B 10, 3342 (1974).

${ }^{3}$ W. E. Spicer, I. Lindau, P. Skeath, and C. Y. Su, J. Vac. Sci. Technol. 17, 1019 (1980).

${ }^{4}$ R. Matz and H. Lüth, Phys. Rev. Lett. 46, 500 (1981).

${ }^{5}$ M. Schubert, Infrared Ellipsometry on Semiconductor Layer Structures (Springer, Berlin, 2005).

${ }^{6}$ N. Marschall, B. Fischer, and H. J. Queisser, Phys. Rev. Lett. 27, 95 (1971).

${ }^{7}$ W. E. Anderson and R. W. Alexander, Jr., Phys. Rev. Lett. 27, 1057 (1971).

${ }^{8}$ K. H. Rieder, N. Ishigame, and L. Genzel, Phys. Rev. B 10, 3804 (1972).

${ }^{9}$ K. Yamamoto, K. Kimura, U. Ueda, H. Kasahara, and T. Okada, J. Phys. C 18, 2361 (1985).

${ }^{10}$ P. Bouchon, F. Pardo, B. Portier, L. Ferlazzo, P. Ghenuche, G. Dagher, C. Dupuis, N. Bardou, R. Hadar, and J.-L. Pelouard, Appl. Phys. Lett. 98, 191109 (2011).

${ }^{11} \mathrm{~F}$. Pardo and Y. Gottesman, "Computation of metallic lamellar structures using a rigorous Maxwell approach with a polynomial approximation of the constitutive equations" (unpublished).

${ }^{12}$ B. B. Varga, Phys. Rev. 137, A1896 (1965).

${ }^{13}$ E. D. Palik, Handbook of Optical Constants of Solids (Academic, New York, 1985).

${ }^{14}$ S. Vassant, F. Marquier, J. J. Greffet, F. Pardo, and J. L. Pelouard, Appl. Phys. Lett. 97, 161101 (2010).

${ }^{15}$ D. Sarid, Phys. Rev. Lett. 47, 26 (1981). 